\title{
Assessing the Reliability and the Expected Performance of a Network under Disaster Risk
}

\author{
Dilek Günneç and F. Sibel Salman, \\ dgunnec@ku.edu.tr, ssalman@ku.edu.tr \\ College of Engineering, Koc University, Istanbul
}

Keywords: network reliability, disaster, dependent link failures, Monte Carlo sampling.

\section{Introduction}

The functionality of infrastructure networks after a disaster is critical for effective disaster response. In a disaster situation, the local and central government agencies and the civil organizations have to dispatch their resources immediately to supply machinery, equipment and relief commodities to the affected areas. As the search and rescue teams work in the field, the injured and dead people will be carried to hospitals. In addition to the time-critical logistics operations carried out by the agencies, some residents will be on the roads trying to leave the affected area while others will try to reach the area to provide humanitarian aid. As a result, the connectivity and consequently the functionality of the transportation networks are vital for the success of the relief operations. In pre-disaster planning stage, it is important to assess the post-disaster performance of the network under most likely disaster scenarios for the purpose of both strengthening the components of the network and for planning the post-disaster logistics activities.

In this study, we seek to measure the reliability and the expected post-disaster performance of a network under disaster risk. In post-disaster response, several nodes in the network would act as supply and demand points. Thus, pairs of origin-destination (O-D) nodes are identified in the network. Then, the connectedness of these pairs of nodes in the post-disaster surviving network becomes critical. We propose a combined measure of reliability, in terms of post-disaster connectedness of the O-D pairs, and expected performance of the transportation network, in terms of expected weighted sum of shortest travel time/distance between the $\mathrm{O}$-D pairs. As the exact computation of this measure is $\sharp \mathrm{P}$-Hard, we propose Monte Carlo sampling based methods to estimate it.

An important aspect of this problem is the nature of uncertainty due to both the disaster and the vulnerability of the components in the network; hence how the network would be affected from a disaster event. We assume that each link of the network will be in one of two states after the disaster: i) operational (it survives), ii) nonoperational (it fails). In most cases, most likely disaster scenarios can be identified. Each disaster scenario is characterized in terms of its intensity and a geographic area of influence. In addition, the vulnerability of the components of the network can be identified, mostly by structural analysis. Hence, one needs to calculate the probability of failure/survival of each link under a given disaster scenario by incorporating all of these factors. One more point to be assessed is whether the links would fail independently or not. In the case of dependent failures, one needs to identify the nature of dependence. We present a framework for handling both independent and dependent failure cases and we illustrate the use of this framework by a case study related to an anticipated earthquake in Istanbul.

\section{Related Work}

Reliability and vulnerability of networks is a well-studied subject. Especially, the increasing terrorism and natural disasters have led to heightened interest in the vulnerability of network systems such as telecommunication, transportation, power, etc., as a matter of national security[1][2]. It has been recognized that it is essential to reinforce these infrastructure network systems for better functionality after any disaster. This requires the ability to determine the critical components of a system. However, how the vulnerability of a network system may be measured is still not clear. This unclarity originates from the fact that even the con- 
cept of vulnerability does not yet have a commonly accepted definition or theoretical basis on which to build [3]. Taylor et al. [1] related vulnerability to the consequences of link failure, irrespective of the probability of failure. While reliability focuses on the connectivity and probability, vulnerability is more closely aligned with network weaknesses and consequences of failure. Ball et al.[4] provided an extensive survey on Network Reliability. Recently, Konak and Smith [5] have reviewed the subject and describe Network Reliability as the ability of a network to continue network services in the case of component failures and pointed out that exact calculation of any general network reliability measure is $\sharp \mathrm{P}$-Hard. Much research has been conducted for reliability calculation including exact methods, theoretical bounds, and simulation. Karger [6] studied all-terminal connectedness using minimum cuts and showed that if the probability that the graph gets disconnected is small, then the Monte Carlo Sampling may estimate this probability within a desired accuracy by means of a Fully Polynomial Randomized Approximation Scheme (FPRAS). In this paper, we focus on Monte Carlo sampling for the estimation of reliability and expected performance. Sampling is an important tool when an intractable number of possible scenarios exist. There are several studies on Monte Carlo simulation-based sample average approximation methods in the literature for stochastic discrete optimization problems [7], stochastic routing problems [8] and stochastic programs with integer recourse [9].

To the best of our knowledge, the studies on network reliability make the assumption that the links of the network fail independently. This is reasonable for some applications such as electric power networks or communication networks in which each link is subject to failure due to intrinsic problems. Even in the disaster management context, when it is difficult to identify the nature of dependency, the independency assumption can be justified. However, often a disaster affects a contiguous region and consequently the failure probabilities of the links depend on each other. An example to that is earthquakes; the links of the transportation network are subject to failure with a probability due to deformation of the highways, building collapses and natural gas line explosions. Given that one of the two connected links has failed, it is more likely that the other one fails as well. Yet, it is already difficult to calculate the reliability under the independency assumption due to the intractable number of possible scenarios. Therefore, a computationally efficient method must be constructed for the dependent failure case. In this paper, we propose a solution approach for estimating the reliability of a network under dependent link failures.

\section{Problem Definition}

The problem considered in this paper is assessing the reliability and travel time of several O-D pairs on a given network $G=(V, E)$, where the links are subject to failure in case of a disaster. Each O-D pair is represented by a commodity $k \in K$ and has a positive weight $r_{k}$, representing estimated population traveling from its origin $O(k)$ to its destination $D(k)$. There is a travel cost $t_{e}$ associated with each link $e \in E$ representing either distance or travel time.

Most likely disaster scenarios are identified, where each disaster scenario is characterized in terms of its intensity and a geographic area of influence. We represent the disaster scenario by a random variable $\omega$ that takes values from the possible set of disaster scenarios, $\Omega=\left\{\omega_{1}, \omega_{2}, \ldots, \omega_{|\Omega|}\right\}$. The probability that scenario $\omega$ occurs is denoted by $\mathrm{P}(\omega)$. Each link of the network will exist in either the operational or the non-operational state after the disaster. We represent the post-disaster state of link $(i, j)$, under any disaster scenario $\omega$, by a random variable $\xi_{i j}$ that takes the value 1 , if link $(i, j)$ is operational after the disaster; and 0 , otherwise. The vector of realizations of the random variables $\xi_{i j}$ over all links $(i, j)$ in $E$, denoted by $\xi=\left(\xi_{i j}\right), \xi \subset\{0,1\}^{|E|}$ induces a subnetwork of $G$, which we refer to as the "surviving network". Let $E(\xi)=\left\{(i, j) \in E: \xi_{i j}=1\right\}$ denote the surviving edges, and $G(\xi)=(N, E(\xi))$ denote the surviving network. The set of all surviving network realizations is denoted by $\Xi=\{1,2,|\Xi|\}$. Cost of a shortest path from $O(k)$ to $D(k)$ in $G(\xi)$ is denoted by $T_{k}(\xi)$. If a particular O-D pair $k$ is disconnected in any network realization, the shortest path length is equal to a penalty cost $M_{k}$ for that pair. The probability of survival of link $(i, j)$ under disaster scenario $\omega$, i.e. $\mathrm{P}\left(\xi_{i j}=1 \mid \omega\right)$ is denoted by $p_{i j}(\omega)$. As mentioned earlier, the likelihood of link failures vary with respect to the intensity and the location of the disaster experienced and the condition of the link components. Thus, this requires a prior analysis of these factors. Still, it may be difficult to obtain a probability distribution for the network realizations. This is because the studies on 
disasters are mostly obliged to use historical data and sufficient data or statistics on link failures are not available in most cases. Therefore, several assumptions have to be made and some parameters should be estimated using available information. We illustrate how this can be done in a case study in Section 4.

In pre-disaster planning, one may be interested in looking into only the most likely worst-case disaster scenario (single scenario case) or a weighted combination of the desired measure over the most likely disaster scenarios (multiple disaster scenario case). Also, reliability and expected travel time are possible measures of interest, concerning either a single O-D pair or a weighted sum over multiple O-D pairs. The measures we are interested in are:

1. O-D Reliability under a single disaster scenario: Probability that $O$ and $D$ are connected given that some disaster scenario $\omega_{j}$ occurs.

2. O-D Reliability under multiple disaster scenarios: $\sum_{j=1}^{|\Omega|} \mathrm{P}\left(\omega_{j}\right) \mathrm{P}\left(O\right.$ and $D$ are connected $\mid \omega_{j}$ occurs).

3. Reliability under a single disaster scenario: Probability that all O-D pairs are connected given that some disaster scenario $\omega_{j}$ occurs.

4. Reliability under multiple disaster scenarios: $\sum_{j=1}^{|\Omega|} \mathrm{P}\left(\omega_{j}\right) \mathrm{P}\left(\right.$ All O-D pairs are connected $\mid \omega_{j}$ occurs).

5. O-D Performance under a single disaster scenario: For O-D pair $k$, and disaster scenario $j$, $\sum_{\xi \in \Xi} \mathrm{P}\left(\xi \mid \omega_{j}\right)\left\{\mathrm{P}\left(O(k)\right.\right.$ and $D(k)$ are connected in $\left.G(\xi) \mid \omega_{j}\right) T_{k}(\xi)$ $+1-\left(\mathrm{P}\left(O(k)\right.\right.$ and $D(k)$ are connected in $\left.\left.G(\xi) \mid \omega_{j}\right) M_{k}\right\}$.

6. O-D Performance under multiple disaster scenarios: For O-D pair $k$, $\sum_{j=1}^{|\Omega|} \mathrm{P}\left(\omega_{j}\right) \sum_{\xi \in \Xi} \mathrm{P}\left(\xi \mid \omega_{j}\right)\left\{\mathrm{P}\left(O(k)\right.\right.$ and $D(k)$ are connected in $\left.G(\xi) \mid \omega_{j}\right) T_{k}(\xi)$ $+1-\left(\mathrm{P}\left(O(k)\right.\right.$ and $D(k)$ are connected in $\left.\left.G(\xi) \mid \omega_{j}\right) M_{k}\right\}$.

7. Performance under a single disaster scenario: For scenario $j$, $\sum_{\xi \in \Xi} \mathrm{P}\left(\xi \mid \omega_{j}\right) \sum_{k \in K} r_{k}\left\{\mathrm{P}\left(O(k)\right.\right.$ and $D(k)$ are connected in $\left.G(\xi) \mid \omega_{j}\right) T_{k}(\xi)$ $+1-\left(\mathrm{P}\left(O(k)\right.\right.$ and $D(k)$ are connected in $\left.\left.G(\xi) \mid \omega_{j}\right) M_{k}\right\}$.

8. Performance under multiple disaster scenarios: $\sum_{j=1}^{|\Omega|} \mathrm{P}\left(\omega_{j}\right) \sum_{\xi \in \Xi} \mathrm{P}\left(\xi \mid \omega_{j}\right) \sum_{k \in K} r_{k}\left\{\mathrm{P}\left(O(k)\right.\right.$ and $D(k)$ are connected in $\left.G(\xi) \mid \omega_{j}\right) T_{k}(\xi)$ $+1-\left(\mathrm{P}\left(O(k)\right.\right.$ and $D(k)$ are connected in $\left.\left.G(\xi) \mid \omega_{j}\right) M_{k}\right\}$.

While the first four measures look at the reliability in terms of connectivity, the next four are combined performance measures, where reliability is also incorporated by means of a penalty cost for disconnectedness.

Exposed to a disaster, the links of a network may fail independently or depending on each other. For manmade intentional disasters (acts of terror) or cases for which the dependencies of link failures may not be characterized, independence assumption may be reasonable. However, for cases in which the disaster affects an area enclosing the network, and the disaster hits certain links with more intensity, it is more reasonable to assume the link failures are dependent. Failure of the links may depend on several factors, both internal and external to the network. The vulnerability of the link itself such as the strength of a bridge is an example to an internal factor whereas, the magnitude and the epicenter of an earthquake may be reckoned as external factors. In addition to these, the consequences of the disaster such as the collapsing of buildings, an explosion or fire may also be the external causes of link failures. As a result, a subset of links tend to act together after the disaster. In that case, the set of links that have dependency are identified leading to mutually exclusive sets $A_{i} \subset E$, for $i=1, \ldots, l$. Each set of links fail independently, while links in a set have dependence among each other. The independent failure case can be represented with singleton sets containing each link separately.

Each link may contain several vulnerable components and each component may withstand different levels of force depending on its structure. This level needs to be translated into parameters, that will be input into a cumulative probability distribution function characterizing the failure probability of the component as a 
function of force on the component. The intensity of a given disaster scenario determines the level of force at different areas, hence the probability of failure of a component under a given disaster scenario is calculated using this function. Survival of the link requires the survival of each component and is calculated from these failure probabilities. This incorporates the spatial factor into the probabilities as links in the same area are subject to the same disaster intensity in a given scenario.

\section{Methodology}

Calculating any of the proposed measures is computationally difficult due to the exponential number of possible surviving network realizations. To overcome this difficulty, we use the Monte Carlo sampling method for both the independent and dependent cases. Here is the pseudo code of the method for the case of independent link failures for a single O-D pair under a single disaster scenario, where $\mathrm{n}$ is the sample size, $\mathrm{c}$ is the number of connected paths, $r e l$ is the reliability of the realization, $S P$ is the set of pre-determined shortest paths and $T_{j}$ is the travel time of path $\mathrm{j}$. The multiple O-D cases under multiple disaster scenarios may be generated similarly.

Pseudo code for independent link failures via Monte Carlo method

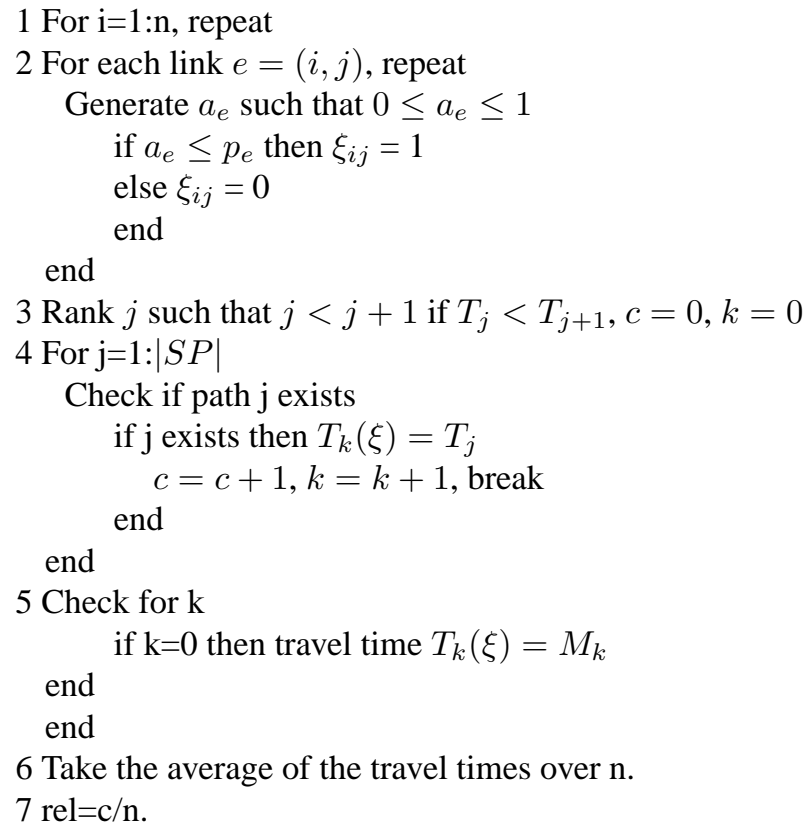

The pseudo code for the case of dependent link failures is given below where $A_{i} \subset E$, represent the mutually exclusive sets for $i=1, \ldots, l$. The dependency is created by generating a single random number to determine the failure of each link in a given set.

Pseudo code for dependent link failures via Monte Carlo method

1 For $\mathrm{i}=1: 1$, repeat

Generate $a_{i}$ such that $0 \leq a_{i} \leq 1$

2 For each link $e=(i, j)_{i}$, repeat

if $a_{i} \leq p_{e}$ then $\xi_{i j}=1$

else $\xi_{i j}=0$

end

end

end 
3 Steps 3 through 7 are the same with the independent case.

\section{Computations}

The proposed methods have been tested in a case study on the urban highway network of Istanbul under earthquake risk. Istanbul is vulnerable to earthquakes due to its seismic-prone location on the North Anatolian Fault; the damage potential is heightened due to its population of over 12 million and commercial/industrial density [10]. It has been reported by Parsons et al. in 2000 [11] that the probability of a major earthquake in Istanbul in the next 30 years is $62.6 \pm 15 \%$. Many studies have been completed on estimating the magnitude and the consequences of possible earthquakes and several most likely earthquake scenarios have been developed [12]. The network considered in this paper is constructed with respect to the two main highways in Istanbul and the bridges/viaducts located on them. The network includes 25 nodes and 30 links. Five O-D pairs have been chosen with respect to the districts with most damage expectations and with higher hospital capacities under the worst case earthquake scenario. Utilizing from previous studies, the sets of edges $A_{i}$ are determined by the measure of Peak Geographic Acceleration (PGA). It is a common tool used by earthquake engineers to measure the earthquake risk of a region. The PGA may be defined as the maximum acceleration experienced by an object in case of an earthquake. Various modeling of PGA distributions of Istanbul are done constituting of four different levels of PGA values[12] with respect to different disaster scenarios. A fragility curve is used to determine the probabilities of failures. The response parameters are chosen as different strength of the links that has been calculated with respect to the structure of the components of the link. The corresponding function values give the fragility of the link with the given property according to the risk each level of PGA entertains. The survival probability of the links are then computed by this fragility curve.

Several sample sizes have been chosen for the Monte Carlo Sampling and the convergence of estimated values has been observed, together with measures of sample variance, and confidence intervals. The convergence of the reliability and performance measures for one of the O-D pairs can be observed in Table 1. The first column gives the increasing number of generated scenarios. The second column gives the cpu time ${ }^{1}$. The third and fourth give the O-D reliability and the O-D performance under a single disaster scenario, respectively, for the independent link failure case. The last three columns present the same values for the dependent case, where dependency sets have been identified based on the five regions of increasing risk values within the affected area, which have been identified in the most likely worst-case earthquake scenario considered.

Table 1: Convergence of Reliability of O-D pair 14-7 for $\mathrm{M}=120$ for the independent and dependent cases

\begin{tabular}{ccccccc}
\hline \hline $\begin{array}{c}\text { no of generated } \\
\text { realizations }\end{array}$ & $\begin{array}{c}\mathrm{cpu} \\
\text { time }(\mathrm{sec})\end{array}$ & $\begin{array}{c}\text { probability of } \\
\text { connectedness }\end{array}$ & $\begin{array}{c}\text { average } \\
\text { travel time }\end{array}$ & $\begin{array}{c}\mathrm{cpu} \\
\text { time }(\mathrm{sec})\end{array}$ & $\begin{array}{c}\text { probability of } \\
\text { connectedness }\end{array}$ & $\begin{array}{c}\text { average } \\
\text { travel time }\end{array}$ \\
\hline 10 & 0.04 & 0.5000 & 68.5476 & 0.02 & 0.4 & 76.4559 \\
\hline 100 & 0.09 & 0.1900 & 99.7284 & 0.04 & 0.57 & 57.9496 \\
\hline 1,000 & 0.51 & 0.2810 & 90.4219 & 0.17 & 0.5290 & 62.4129 \\
\hline 10,000 & 4.83 & 0.2914 & 89.2341 & 1.38 & 0.5431 & 60.8780 \\
\hline 100,000 & 47.44 & 0.2938 & 89.0514 & 5.12 & 0.5512 & 59.9940 \\
\hline $1,000,000$ & 466.55 & 0.2941 & 89.0106 & 51.01 & 0.5503 & 60.0952 \\
\hline
\end{tabular}

\section{Conclusion}

The computational results of the case study for the independent failure case and preliminary experiments for the dependent link failure case support that reliability and performance of a network of realistic size can be estimated with high accuracy in moderate cpu time with the proposed Monte Carlo simulation method.

\footnotetext{
${ }^{1}$ All of the computations have been carried on the PC with $2 \times 2.8 \mathrm{GHz}$ Xeon Linux processor and 5 GB RAM memory with the sampling algorithm implemented in Matlab 7.0.
} 


\section{References}

[1] Taylor, M. W. P., D'este, G. M., "Concepts of Network Vulnerability and Applications to the Identification of Critical Elements of Transport Infrastructure," 26th Australian Transport Research Forum Wellington, New Zeland, 1-3 October 2003.

[2] Moselhi, O., Hammad, A., Alkass, S., Assi, C., Debbabi, M., Haider, M., "Vulnerability Assessment of Civil Infrastructure Systems: A Network Approach," 1st CSCE Specialty Conference on Infrastructure Technologies, Management and Policy, Toronto, Ontario, Canada, 2-4 June 2005.

[3] Jenelius, E., Petersen, T., Mattson, L. G., "Importance and Exposure in Road Network Vulnerability Analysis," Transportation Research Part A, 40, pp. 537-560, 2006.

[4] Ball, M. O., Colbourn, C. J., Provan, J. S., "Network Reliability," Handbooks in OR \& MS, 7, pp 673-762, 1995.

[5] Konak, A., Smith, A. E., "Network Reliability Optimization," Handbook of Optimization in Telecommunications, Resende, M. G. C, Pardolos, P. M., XXXII Springer Science, Business Media, New York, pp 735-760, 2006.

[6] Karger, D. R., "A Randomized Fully Polynomial Time Approximation Scheme for the All-Terminal Network Reliability Problem "' Proceedings of the Twenty-Seventh Annual ACM Symposium on the Theory of Computing, ACM Press, pp. 11-17, 29 May-1 June 1995.

[7] Kleywegt, A. J., Shapiro, A., Homem-De-Mello, T., "The Sample Average Approximation Method for Stochastic Discrete Optimization," SIAM, 12, No.2, pp. 479-502, 2001.

[8] Verweij, B., Ahmed, S., Kleywegt, A. J, Nemhauser, G., Shapiro, A., "The Sample Average Approximation Method Applied to Stochastic Routing Problems: A Computational Study," Computational Optimization and Applications, 24 , Issue 2-3 pp. 289-333, 2003.

[9] Ahmed, S., Shapiro, A., "The Sample Average Approximation Method for Stochastic Programs with Integer Recourse," Published electronically in Optimization Online, 2002.

[10] http://www.worldbank.org.tr Istanbul Seismic Risk Mitigation and Emergency Preparedness Project.

[11] Parsons, T., Toda, S., Stein, R.S., Barka, A., Dieterich, J.H ., "Heightened Odds of Large Earthquakes Near Istanbul: An Interaction-Based Probability Calculation,” Science, 288, pp 661-665, 2000.

[12] "The Study on A Disaster Prevention / Mitigation Basic Plan In Istanbul Including Microzonation In The Republic of Turkey", Japanese International Cooperation Agency, Local Municipality of Istanbul, Final Report Volume V, September 2002. 